$\mathrm{RHIC}-\mathrm{PH}-6$

\title{
QUARK-GLUON PLASMA \\ AND \\ RELATIVISTIC ION COLLISIONS
}

\author{
L. Van Hove \\ Brookhaven National Laboratory \\ November 1984
}

$\theta$ 
QUARK-SLUON PLASTID AND

RELATIVISTIC ION COLLISIONS.

L. VAN HoVE

A labwide lecture and two seminars at Brookhaven National Laboratory

(November 1984) 
Atom

Nucleus

Nucleon

$880 \%$

@is

- electron

o nucleons

(lepton family)

(hadron family)

- quarks

(quark family)

Strong interaction: acts on quarks,

Electroweak interactions: act on quarks and leptons, are mediated by photons and Weak bosons.

$$
\left(W^{ \pm}, z^{0}\right)
$$

Leptons, photons, weak bosons, hadrons can exist as isolated particles. quarks and gluons don't (confinement property): 
Confinement - Hadronization

$$
\begin{aligned}
& \left.=10^{-13} \mathrm{~cm}\right] \stackrel{\mathrm{m}}{1}
\end{aligned}
$$

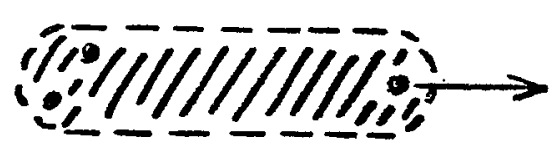

IIIIIII/ colour electric field

QCD flux tube (also called string).

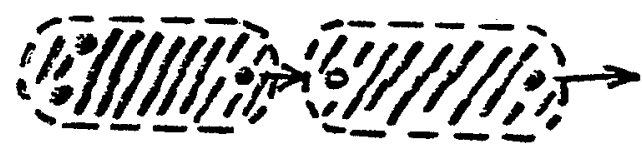

Hadronization by $q \bar{q}$ pair creation.

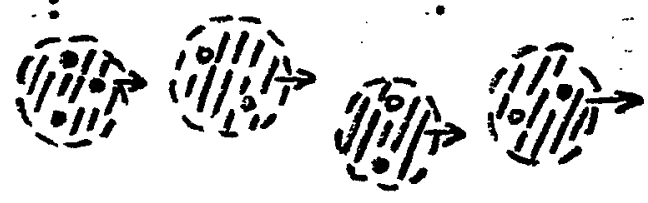

String tension $\sim 1 G \times 1 / \mathrm{fm}$

For comparison, "liberation" of electron:

$=10^{-8} \cos I$

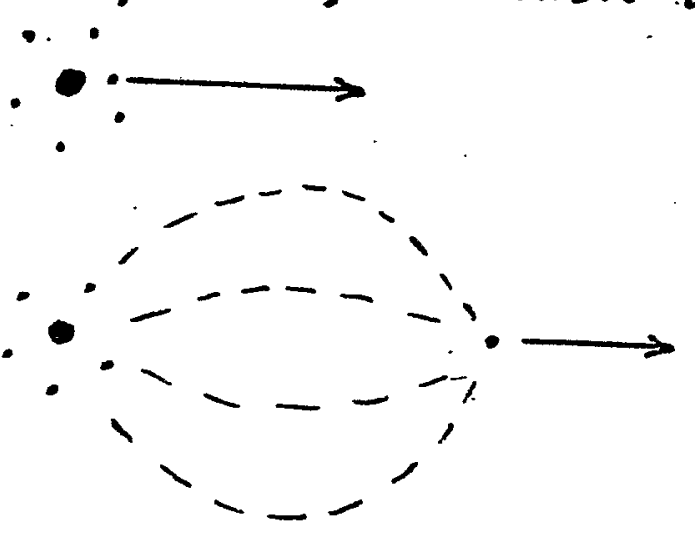

Electric field 
Theoretical prediction of a ness state of matter,

13

the "QUARK- SLUON PLASTA"(also called" quark matter").

- Constitution of hadronic matter according $t o$ QUANTUM CHROMODYNABicS (QCD); a um-ablican gauge theory based on $S U_{3}$ (colour qu. nbs) Spinor fields $\rightarrow$ QUARTS, sc triplets, 5 (6.) "flavours" gauge fields $\rightarrow$ GLUONs, sO octets, no further int. qu. ht.

- CONFINEBENT property: hadronic systems in vacuum form $S U_{3}$ singlets (colourless")

Examples: baryon $=(999)_{\text {ringlet }}$, meson $=(9 \overline{9})$ singlet?

$\because \because \because 0 ; \quad$ where $q, \bar{q}$ may Be "dressed by a "sea". of $q \bar{q}$ pairs and gluons.

- nucleus is also sUr singlet, containing SA quarts and perhaps more ( $9 \overline{9}$ pain).

q, $\bar{q}$ distribution in nuclei is not the one in unseteons folded with Fermi distribution (EMCeffect)

- At low density, hadronic matter forms HADRON GOS.

- Single hadrons occupy finite volume, $\geqslant 1 \mathrm{fm}^{3}$.

- What about very dense hadronie matter as must aces i) when nuclear matter is highly compressed (dense stellar abjectly is jat high temperature, high density of hadrons being created by thermal agitation (early Universe). Hadrons must then coalesces is to a dense, continuous fluid of g's, i's and givens, the QUARK-TLUOAN PLASHA. 
i) Compress nuclear matter by factor 20 :

14 que art density a $20 \times 3 \times 0.17 \mathrm{fm}^{-3}-10 \mathrm{fm}^{-3}$ ii) Heat matter to To 500 Meld o $\sigma_{x} 10^{12} K$ : An ideal pion gas would have $\sim 6$ pions $/ \mathrm{fm}^{3}$, Lie., a $q+\bar{q}$ density $\sim 12$ for $^{-3}$

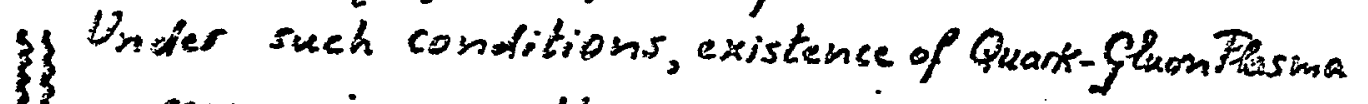
stems inescapable.

- Can this ness state of matter be produced on carts? Perhaps in ultra relativistic nuclear collisions

- 3 classes of problems, all affected by large uncertainties:

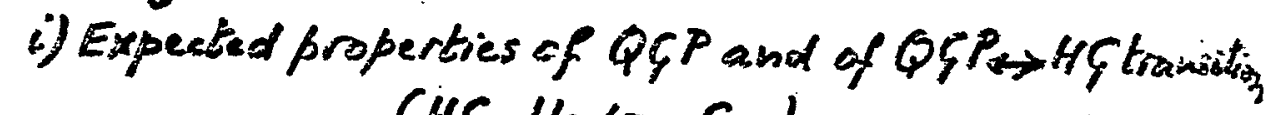
(HG= Hadron Gas).

ii) Compression and heating in nuclear collisions. (iii) Signals for QSP formation in .

- first theoretical work, great quantitative uncertainties. - Severe lack of data hampers. Work; expertmental programmes to start soon at Brookhaven (15 GeV/nuelion up to 325 ) and CERN (225 GeV/usateon up to ${ }^{10} \mathrm{O}$ ).

- Impossibility to measure "colour conductivity."

RHiC: colliding beams up to gold (" ${ }^{197}$ Au) up to 100 geV/nucleon. (RHiC: Relativistic Heavy Ion Collider) 


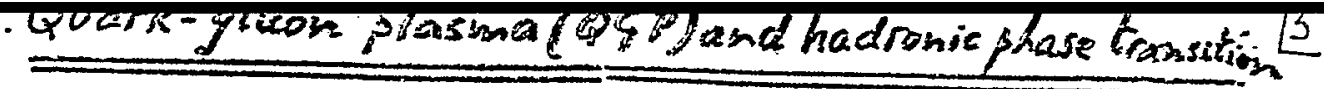

On s expects 2 phase transitions, but they may coincide:

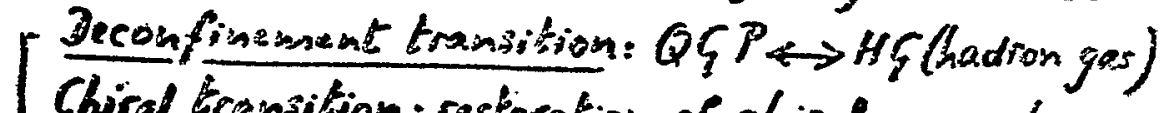

I Chiral transition: restoration of chiral symmetry

(from $\left\langle\psi_{9} \bar{F}_{9}\right\rangle \neq 0$ to $\left\langle\psi_{q} \bar{y}_{9}\right\rangle=0$ )

Theoretical works follows 2 approaches:

Continuum approach:

describe tI G from hadron phenomenology

"QSP by ideal gas with perturbative and "plasmon corrections" based on QCD.

(complexity of collective effect!)

Lattice approach: thermodynamics of QCD in lattice approximation by Monk e Carlo integration.

- Pure SU, gauge field (self-interacting gluons):

first order phase transition, large latent heat.

( $\varepsilon_{Q \varphi P} \gg \varepsilon_{H T}$ at transition, $\varepsilon=$ energy density)

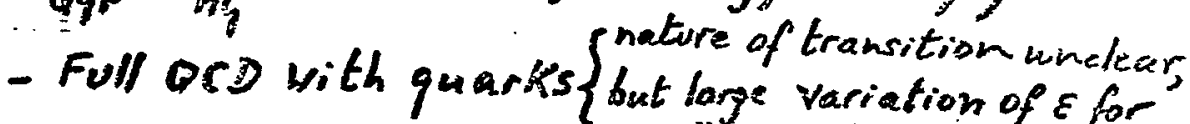
small variation of $T^{2}$, for

Possible phase diagram ( $\nu=$ net quart's density, $\mu=$ quark chemical
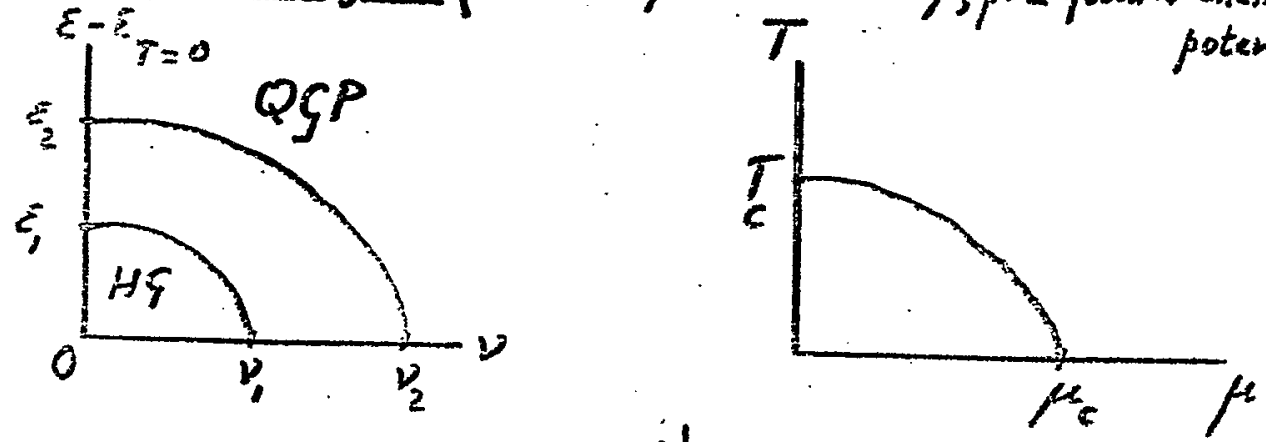

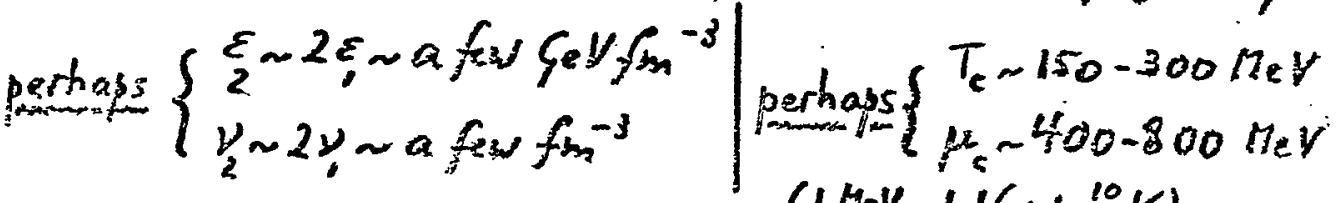

$$
\begin{aligned}
& \left(1 \mathrm{Hel}=1.16 \times 10^{10} \mathrm{~K}\right)
\end{aligned}
$$


Uetrarelativistie nuclear collisions.

6

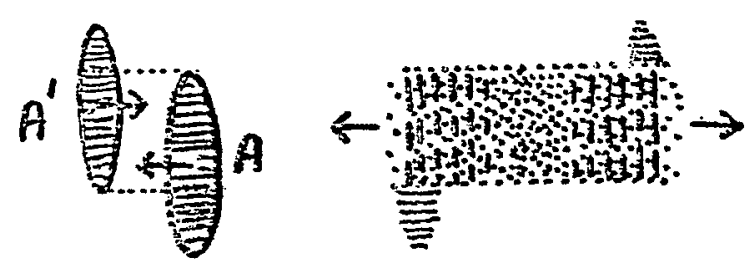

三" spectators"

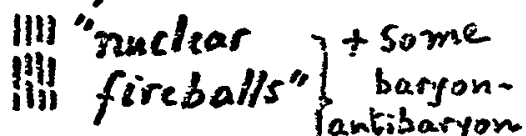

i: mesons pair erection

Longitudinal rapidity: $y=\operatorname{arctin} v$ Net baryon number: $B$

Pong.
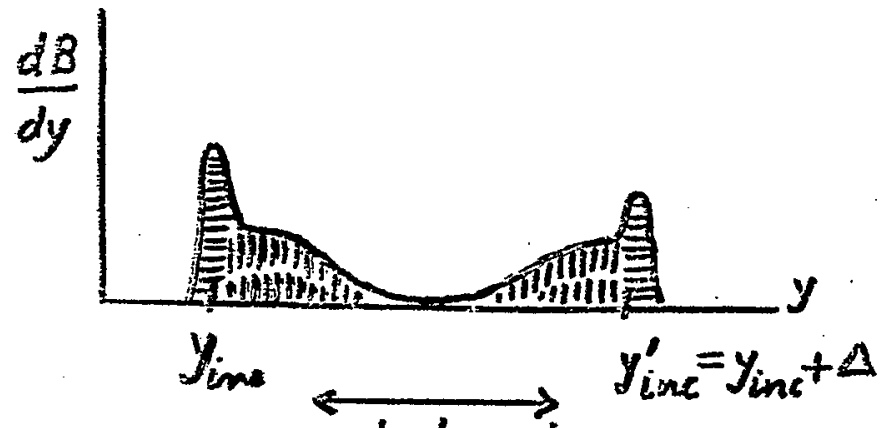

Central region

$\underset{\text { fragmentation }}{\stackrel{\sim}{\sim} \underset{\text { regions }}{\sim}}$

$\frac{d E}{d y}$

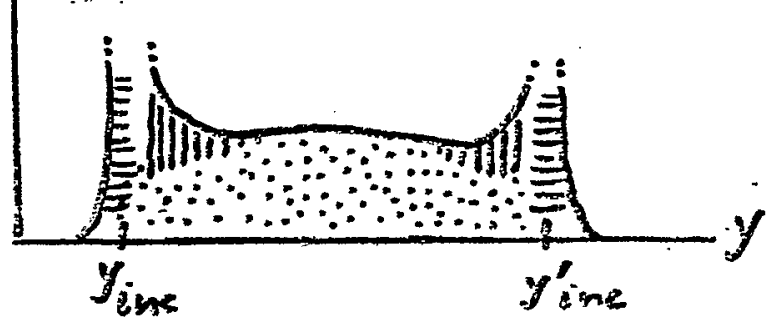

$\left(\begin{array}{l}\Delta=\text { for } \\ E_{l a b}=200 \mathrm{GV} / \mathrm{AV}\end{array}\right)$

d.

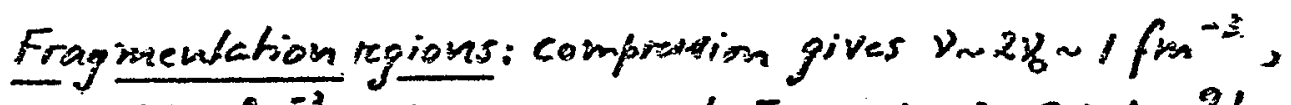

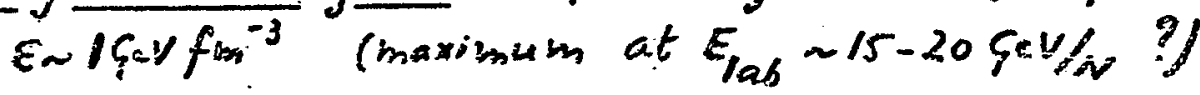

Central region: perhaps $E_{2} \geqslant 2 \mathrm{~F}_{2} \mathrm{H} \mathrm{fm}^{-3} \mathrm{at} E_{\mathrm{lab}} \geq 200 \mathrm{GeV} / \mathrm{N}$ (Keeps growing with energy)

But:FluCTUATIONS cash Ret? 
Signals for Parma formation.

$t$

"Traditional" signals:

- direct $e^{*} e^{-}$ind $\mu^{*} p^{-}$pair production

- siret photon production:

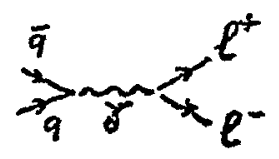

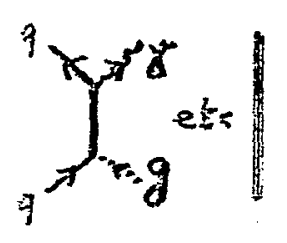

to b. calculated in plasma as fer of T Tend $\nu$, spacetime distribution of $T_{3} \nu$ needed.

production in absence of plasma poorly know in.

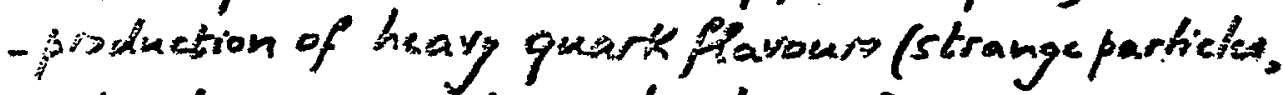

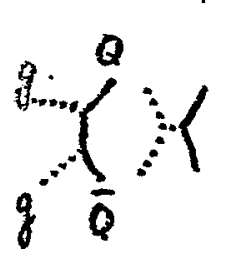
charmed particles too heavy? not in equilibrium in plasma, mainly produces in hot plasma, probably more abundant than without platina (production by gluons mainly).

Signals of phase transition with Page Patent heat:

- $\left\langle\beta_{z}\right\rangle-$ multiplicity correlation (observed in central

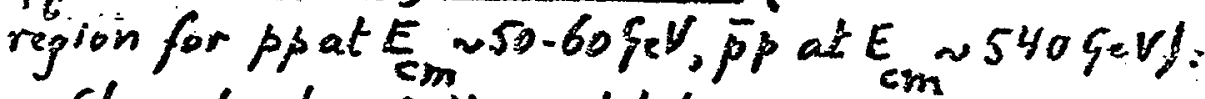

Characteristic of thermal betavieser:

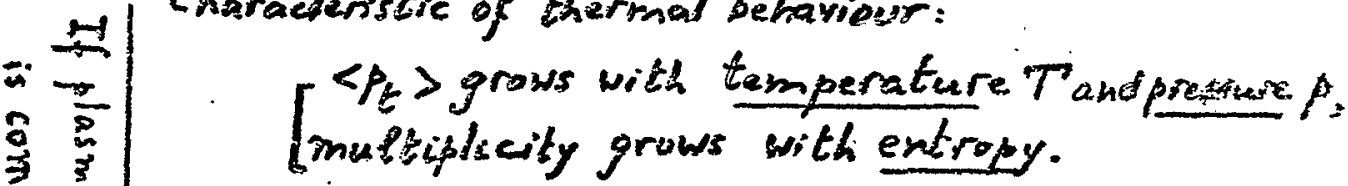

But phase transition with large latent heat gives large increase of entropy without increase of $T_{2} A$ This might show as an anomaly in $\left\langle\beta_{t}\right\rangle-$ malt. came.

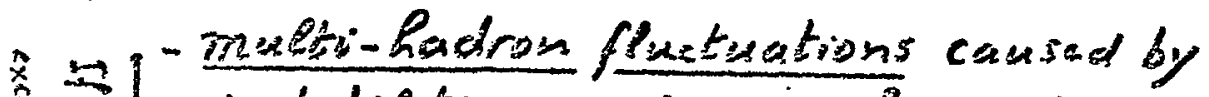

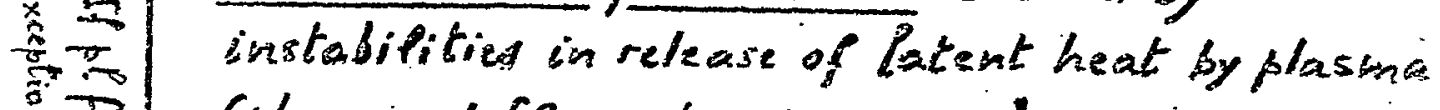
(plasma deflagration) s. Cosmic ray evidence?

$$
Q G P \text { if } H \rightarrow
$$

Shock front 
Signals for plasma formation (in pictures)

18

i) electromagnetic radiation emitted by plasma
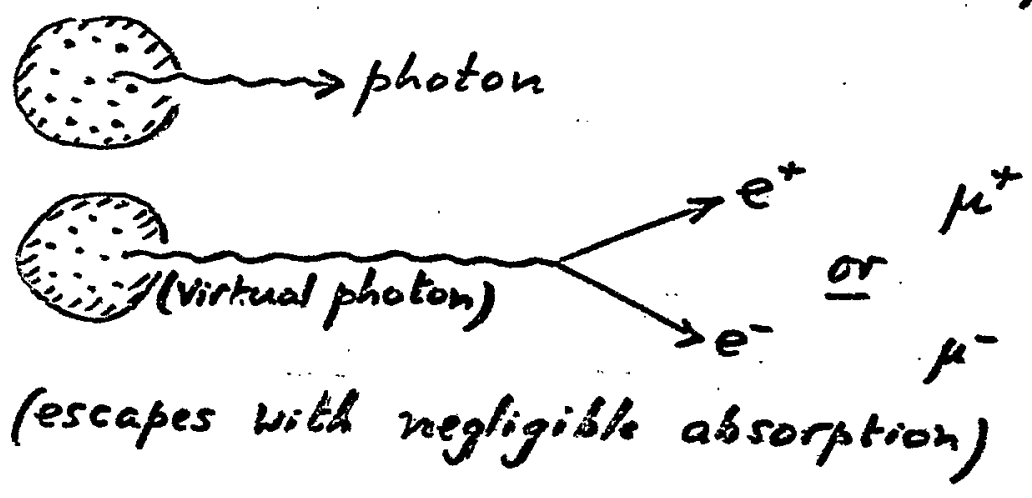

ii) strange quark pairs formed in plasma, emitted as strange hadrons

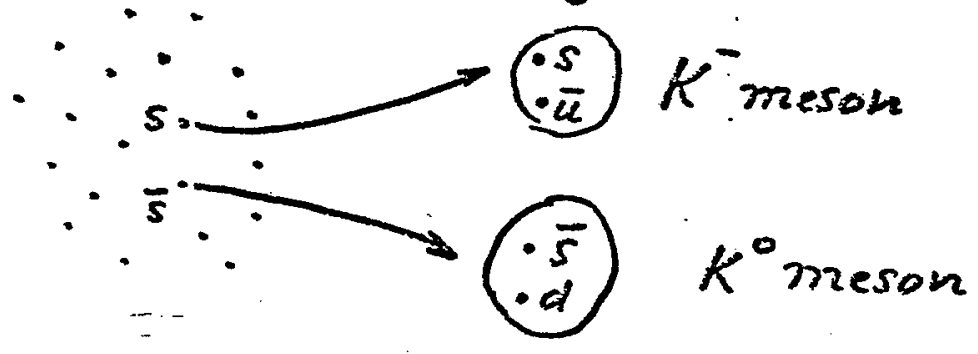

iii) sudden liberation of latent heat at transition

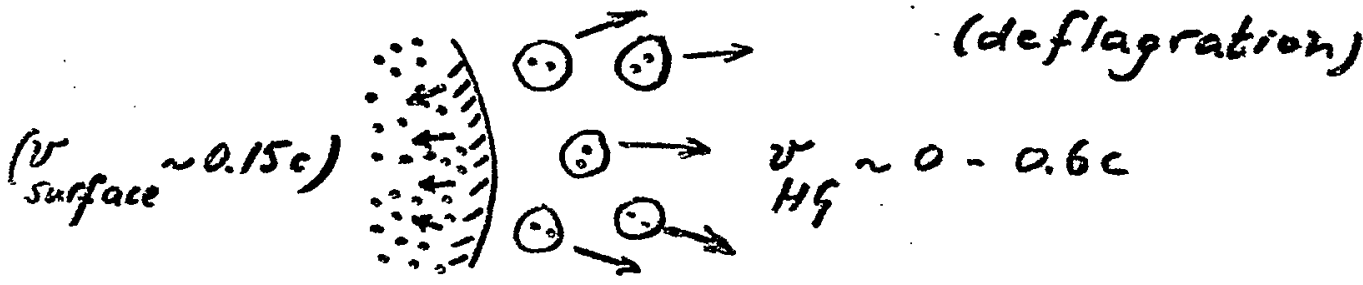

$$
\begin{aligned}
& \text { Plasma } \\
& \text { Hadrons } \\
& \text { high energy contents } \rightarrow \text { high transverse motion } \\
& \text { "entropy " } \rightarrow \text { " multiplicity }
\end{aligned}
$$


$\therefore \quad$ Proposed 5esinario fer

19

Hadronciation of expanding droplet of $Q$ S Plasma

- $\delta$ : nearest neighbour distance between $Q C D$

"partons" (= quarts, amtiquarks, gluons)

- For $b<1$ fin colour confining fields lie QCogauge fields) cover all space in plasma.

- When $d \geq 1$ fin the fields collapse into strings (=flux tubes/separated by vacuum (non-pertweb. QCD vacuum)
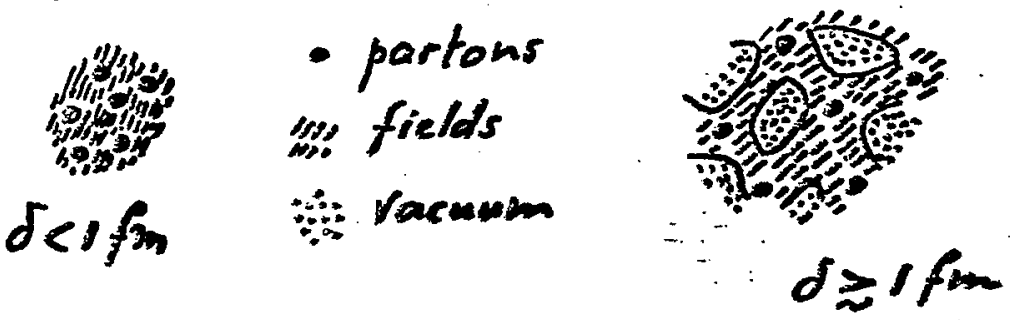

- Some strings break by parton pair creation (light quarts)

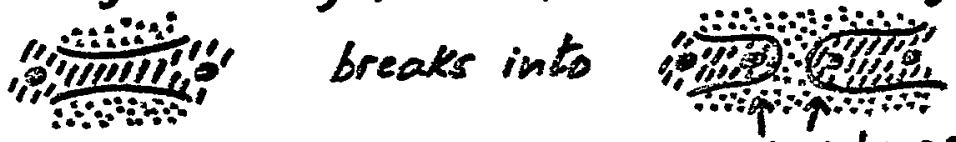

$$
\begin{aligned}
& \text { newly ereatod } \\
& \text { parsons }
\end{aligned}
$$

- A small expanding droplet breaks into smaller opes and string tension stops expansion of the pieces
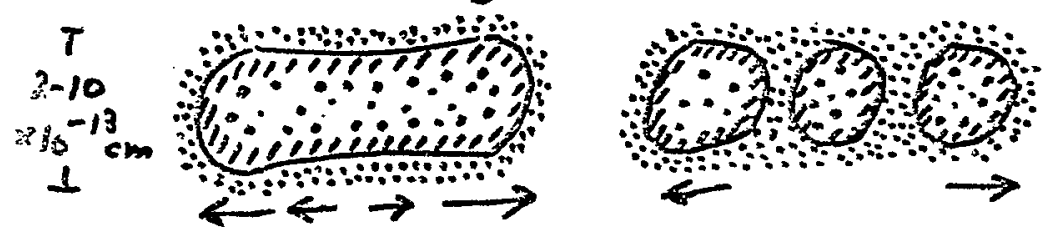

Time scale

$$
10^{-22}-10^{-20}
$$

- Non -expanding plasma droplet can "deflagrate." by emitting a flow of hadron gas into vacsession.

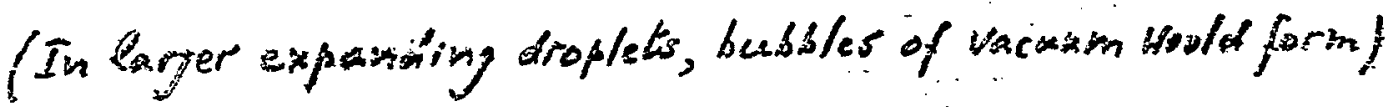


Heavy hadrons of high spin: $J=\alpha^{\prime} m^{2}+c^{t}$

$$
\alpha^{\prime} \simeq 1 \operatorname{GeV}^{-2}
$$

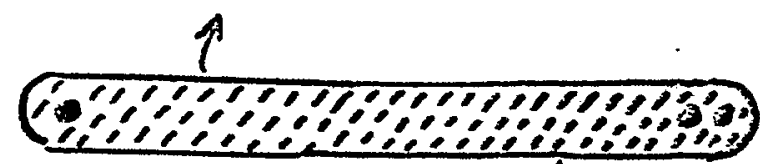

- String of length $L$, diameter $D=1, \mathrm{mon}=\frac{1}{200 \mathrm{kaV}}$

- Mass of non-rotating string $\angle \sigma$

$$
\sigma=\frac{\pi}{4} D^{2} T_{0} J_{0}
$$

- Rotating string, endpoints velocity $c=1$

mass: $\frac{\pi}{2} L \sigma=m$

spin: $\frac{\pi}{8} L^{2} \sigma=J$

$$
T=\frac{m^{2}}{2 \pi \sigma}, \alpha^{\prime}=\frac{1}{2 \pi \sigma}
$$

Using $D=1, \operatorname{m}, \alpha^{\prime}=1 \operatorname{se} V^{-2}$ one finds

$$
\sigma=0.16 \mathrm{GeV} V^{2}, T_{0}^{5}=10^{-2} G \mathrm{~g} V^{4}=1.25 \frac{G \mathrm{GV}}{\mathrm{fmos}^{3}}
$$

An ideal gas of gluons and light greats $(u, q)$ at $T_{0}=150 \mathrm{HeV}$ would have $T_{0}=1 \mathrm{GaV} / \mathrm{fm}^{*}$. 
Colour fields in expanding plasma

111

\& quarks and antiquaries

- gluons

HIIIII colour fields

$\because: Q D$ vacusem
If m $=10^{-13} \mathrm{~cm}$

if m
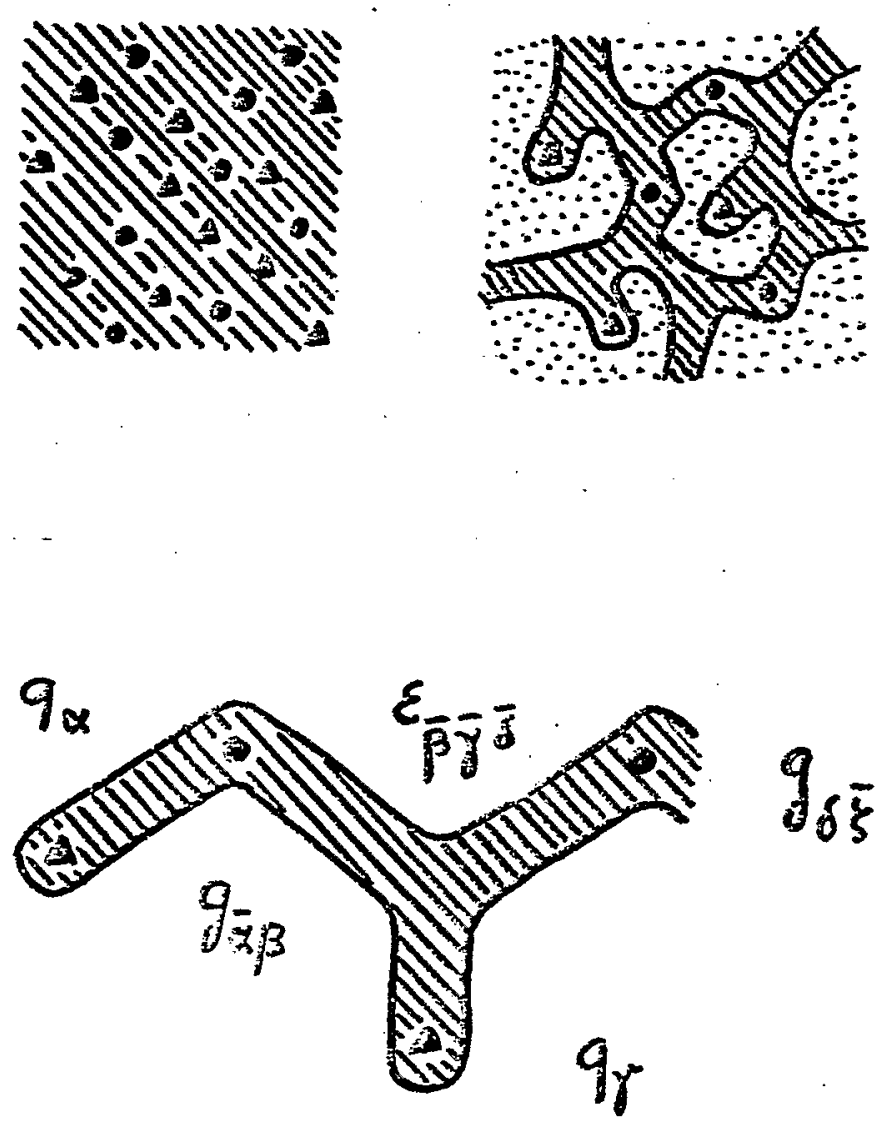
Can the Ques: Gluon Plasma deflagrate?

112

Can it liberate its latent heat by emitting a flow of Hadron $\zeta a{ }^{2}$ ?

Picks: in QGPrest frame:

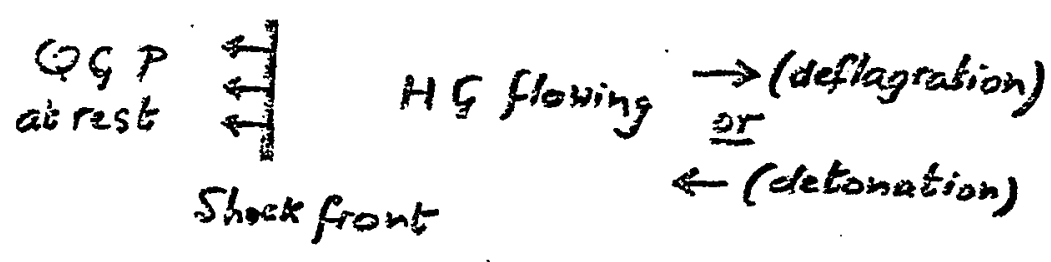

Dictate in rest frame of shock front:

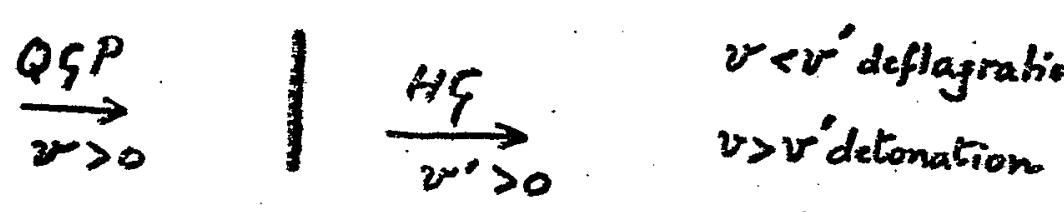

Question = is this possible with in creese of entropy? [If entropy would deccecise, inverse process ( $\left.v<0, v^{*}<0\right)$ could take place: compression of HS into $Q \zeta P 7$

Elegy conservation: $\frac{v(\varepsilon+\beta)}{1-v^{2}}=\frac{v^{\prime}\left(\varepsilon^{\prime}+p^{\prime}\right)}{1-v^{2}} \rightarrow v v^{\prime} \geqslant 0$

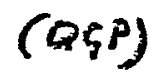

(HG)

$$
\begin{aligned}
& \varepsilon+\beta>\varepsilon^{\prime}+\beta^{\prime} \rightarrow \mid \text { w }|<| \sim \mid \text { like deflagration }(0<v<v) \\
& \text { or compression }(\omega<-v<-v)
\end{aligned}
$$

+ Momentum cons.: $\frac{v^{2}}{1-v^{2}}=K \frac{\varepsilon^{\prime}+\beta}{\varepsilon+p}, \frac{v^{\prime 2}}{1-v^{2}}=k \frac{\varepsilon+\beta^{\prime}}{\varepsilon^{\prime}+p^{\prime}}, K=\frac{p-\beta^{\prime}}{\varepsilon-p-\varepsilon^{\prime}+\beta^{\prime}}$

Baryon na cons.: $\nu^{2} \frac{\varepsilon+\beta}{E+p}=\nu^{\prime 2} \frac{\varepsilon+p^{\circ}}{\varepsilon^{\prime}+p^{\prime}}$

Entropy increase: $\left\{\begin{array}{l}\operatorname{\nu and} \nu^{\prime} \neq 0: \sigma / \nu \leqslant \sigma^{\prime} / \\ \nu=\nu^{\prime}=0: \frac{\sigma^{2}}{\sigma^{\prime 2}} \leqslant \frac{(\varepsilon+\beta)\left(\varepsilon+\beta^{\prime}\right)}{\left(\varepsilon^{\prime}+\beta\right)\left(\varepsilon^{\prime}+\beta^{\prime}\right)} \leftrightarrow \frac{\tau^{\prime 2}}{\gamma^{2}} \leqslant \frac{\varepsilon+\beta^{\prime}}{\varepsilon+\beta} \frac{\varepsilon^{\prime}+\beta^{\prime}}{\varepsilon^{2}+\beta}\end{array}\right.$ 
Grashical treat unent' of $\nu_{x} \nu^{\prime}=0$ casa e eniropy inerease $T T_{0}^{\prime} \leqslant T^{\prime} T_{0}$

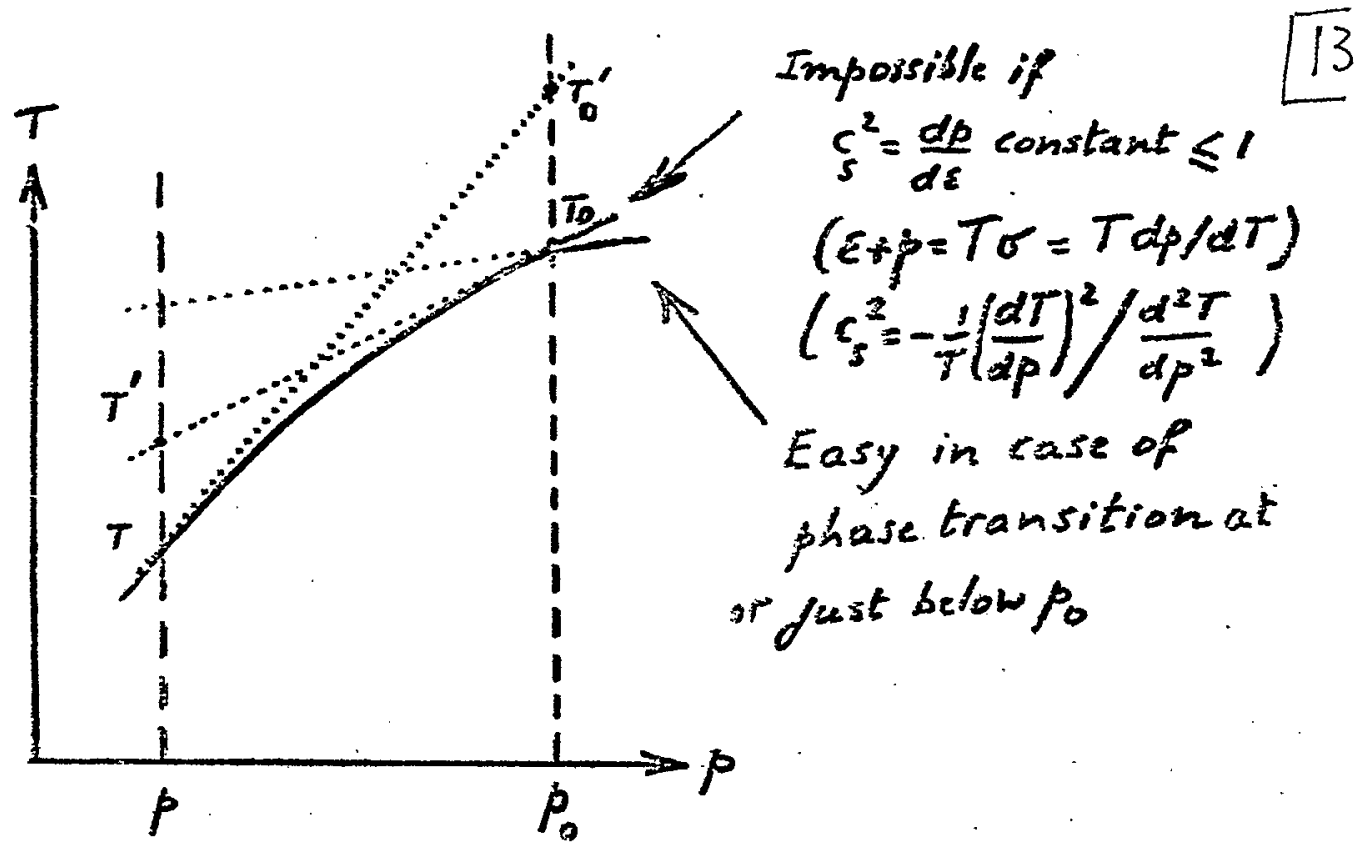

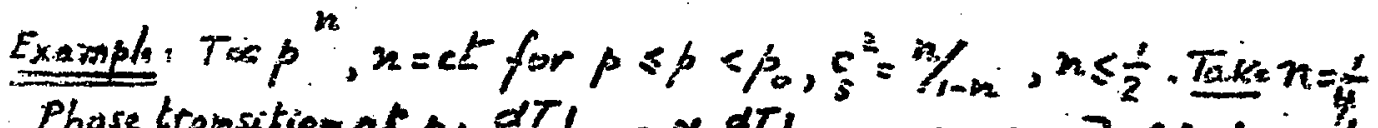

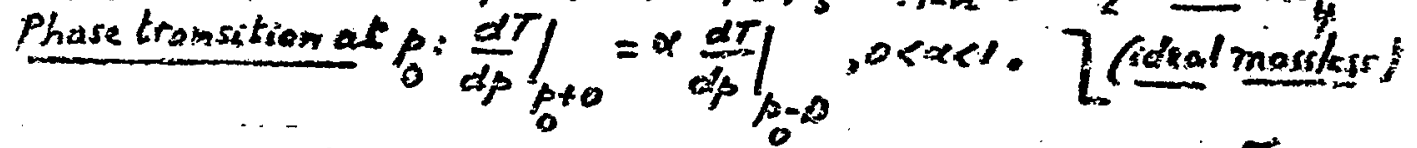

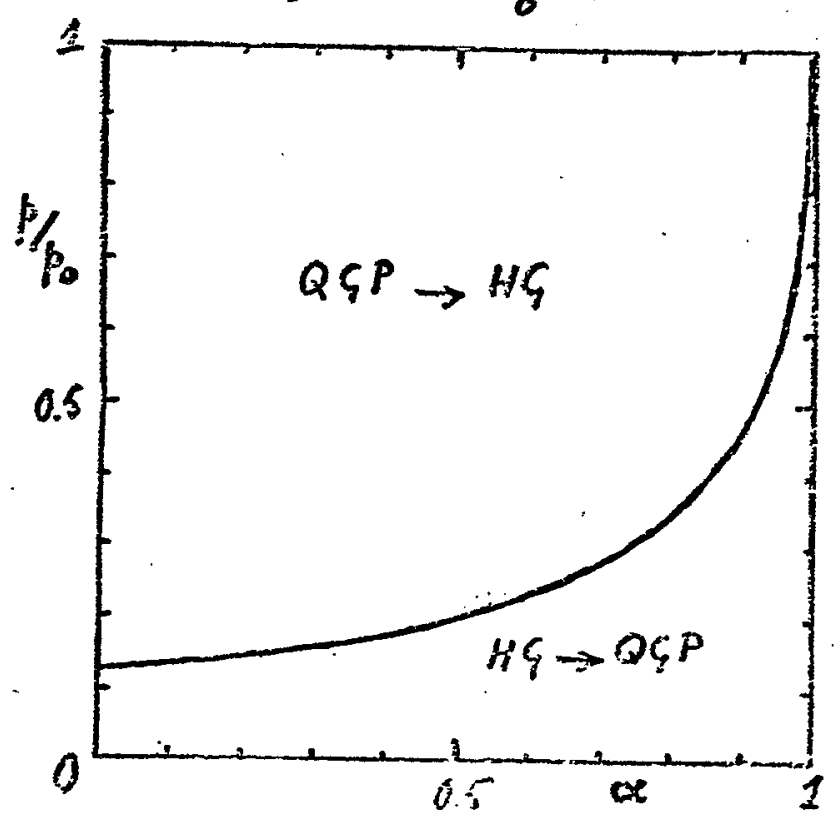

$$
\left(\begin{array}{l}
\alpha=\frac{\sigma_{\text {gas }}}{\sigma_{\text {plasme }}} \\
\text { at: tramsition }
\end{array}\right)
$$


$-(14$

$$
\left.\begin{array}{l}
F^{g} \text { :entropy flux in gas } \\
F^{\text {ip g }} \text { :entropy flex in plasma }
\end{array}\right\} \text { in rest frame of fro ut. }
$$

First order trans, $\alpha=\sigma_{g} / \sigma_{\beta}=0.5$ at transition.

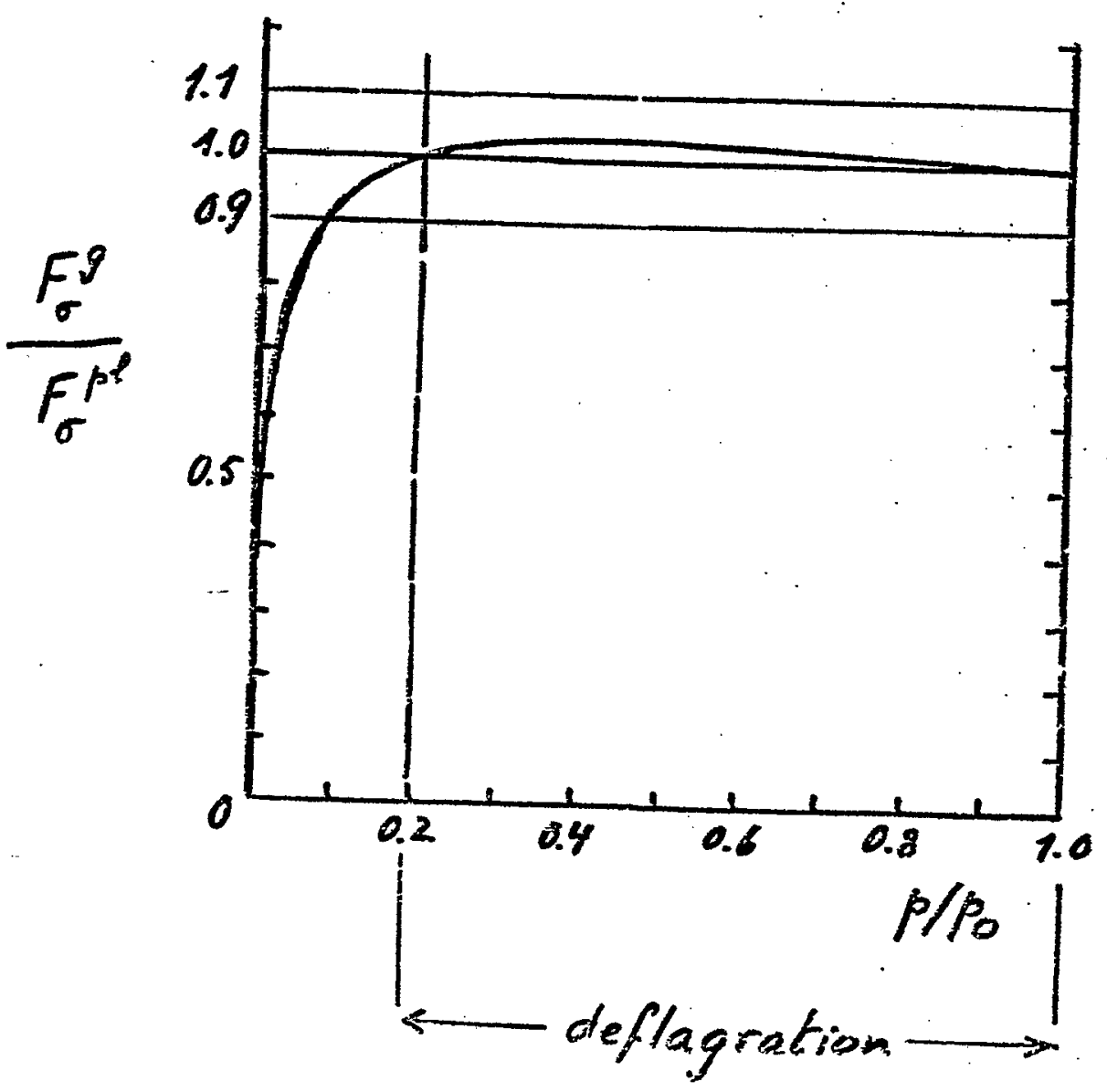



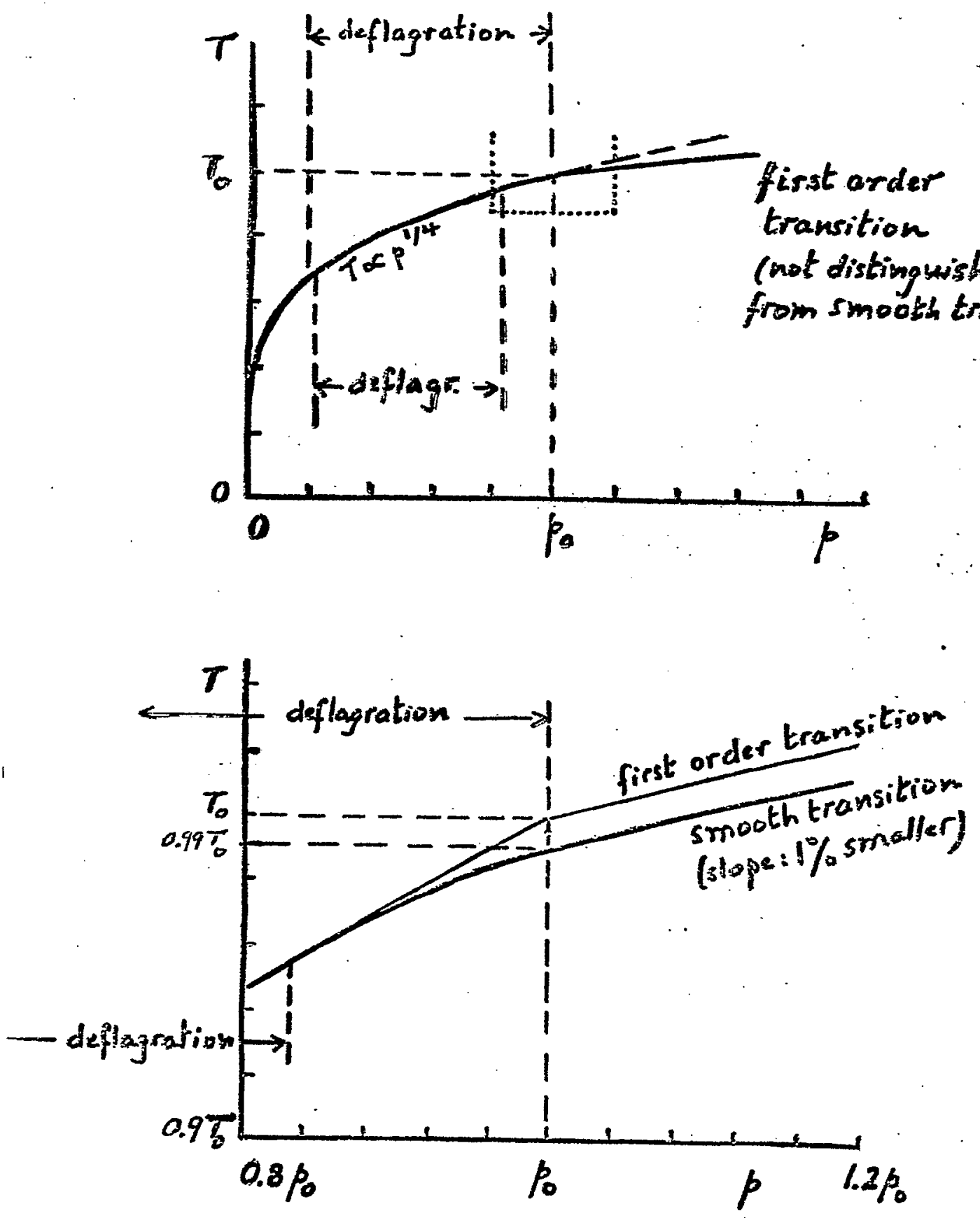

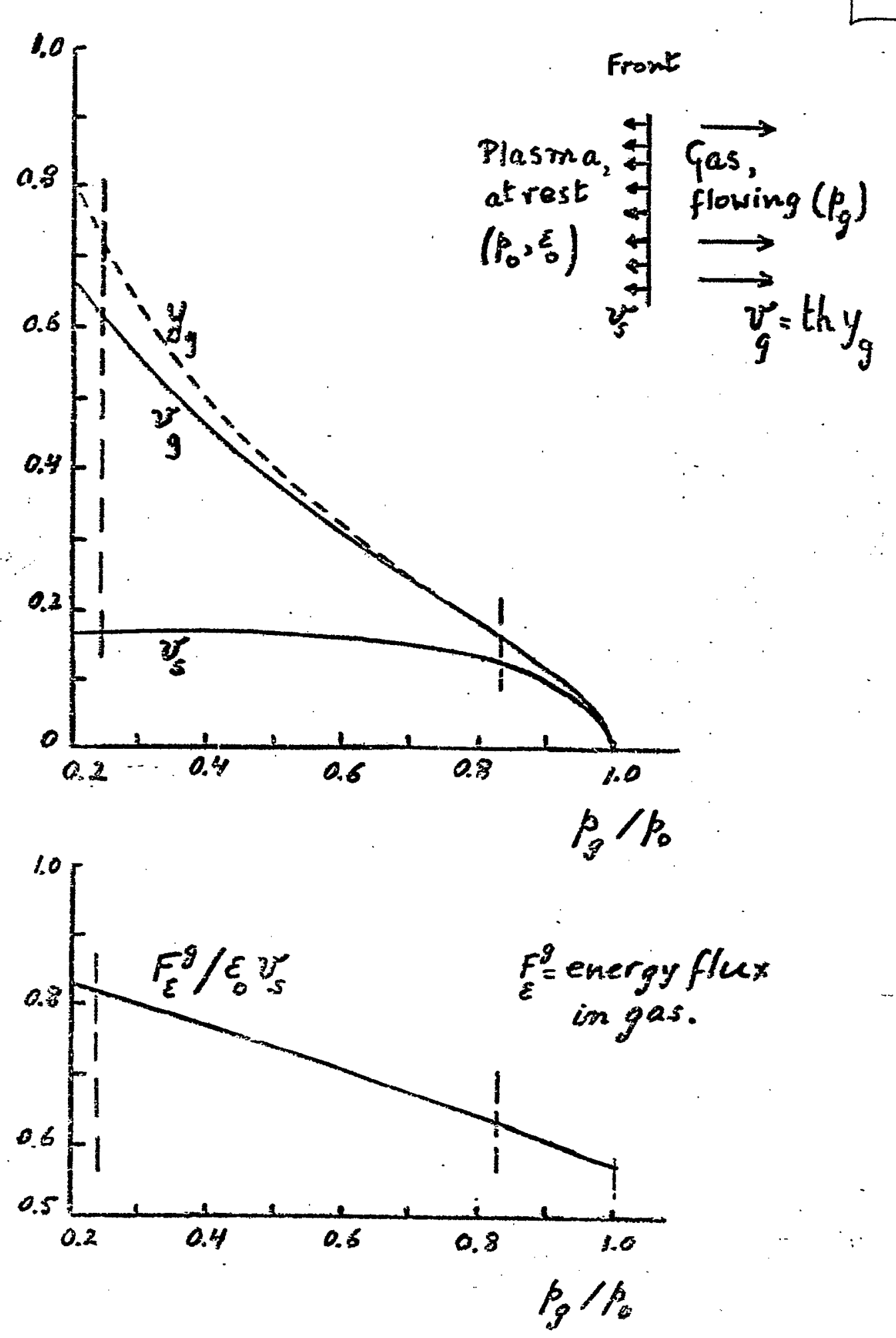
Discussion of energy flax $F_{\varepsilon}^{9}=\left(\xi \times p_{g}\right) \lg _{g}\left(1-r_{g}^{2}\right)^{-1}[17$

- Order of magnitude $\frac{3}{4} * \frac{1}{6} \times \varepsilon_{0}=\frac{1}{8} \varepsilon_{0}$

$$
\frac{f_{\varepsilon}^{g}}{\xi v_{s}} \longrightarrow_{v_{s}}
$$

- What would it be if all quests, andiqueartes and gluons flying toward surface would cross it without energy loss?

$$
\begin{aligned}
& \frac{1}{2} \times \frac{1}{2} \times \frac{\varepsilon}{0}=\frac{1}{4} \varepsilon_{0} \\
& b \frac{\cos \theta}{b a l f} \text { of the quanta }
\end{aligned}
$$


Experimental consequences.
If plasma droplet (possibly after breaking as)

hadronizes by deflagration,

resulting rapiotify distribution of hadrons

show lo show maxima at rapidities of droplets

$\leftarrow$ Plasma $\rightarrow$ Expanding droplet

breaks into

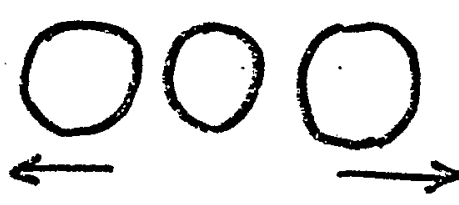

non-expanding droplets

$\frac{d n}{d y}$

resulting maxima

OR in hadron rat -distr.

y (long rapidity)

Expected Width of maxima $\sim 1 \div 1.5$

- The other signals for plasma formation

(direct dilaptons ant photons, strange hadrons)

should be concentrated in these maxima.

- Hadrons from plasma should have po somewhat

lagger than normal, with broad but fluctuating azimuthal distribution. 
THE HADRONIC PHASE TRANSITION IN THE EARLY UNIVERSE.

L. VAN HOVE

A colloquium Lecture at Bisothaven National Laboratory (November 1984) 
$-1$

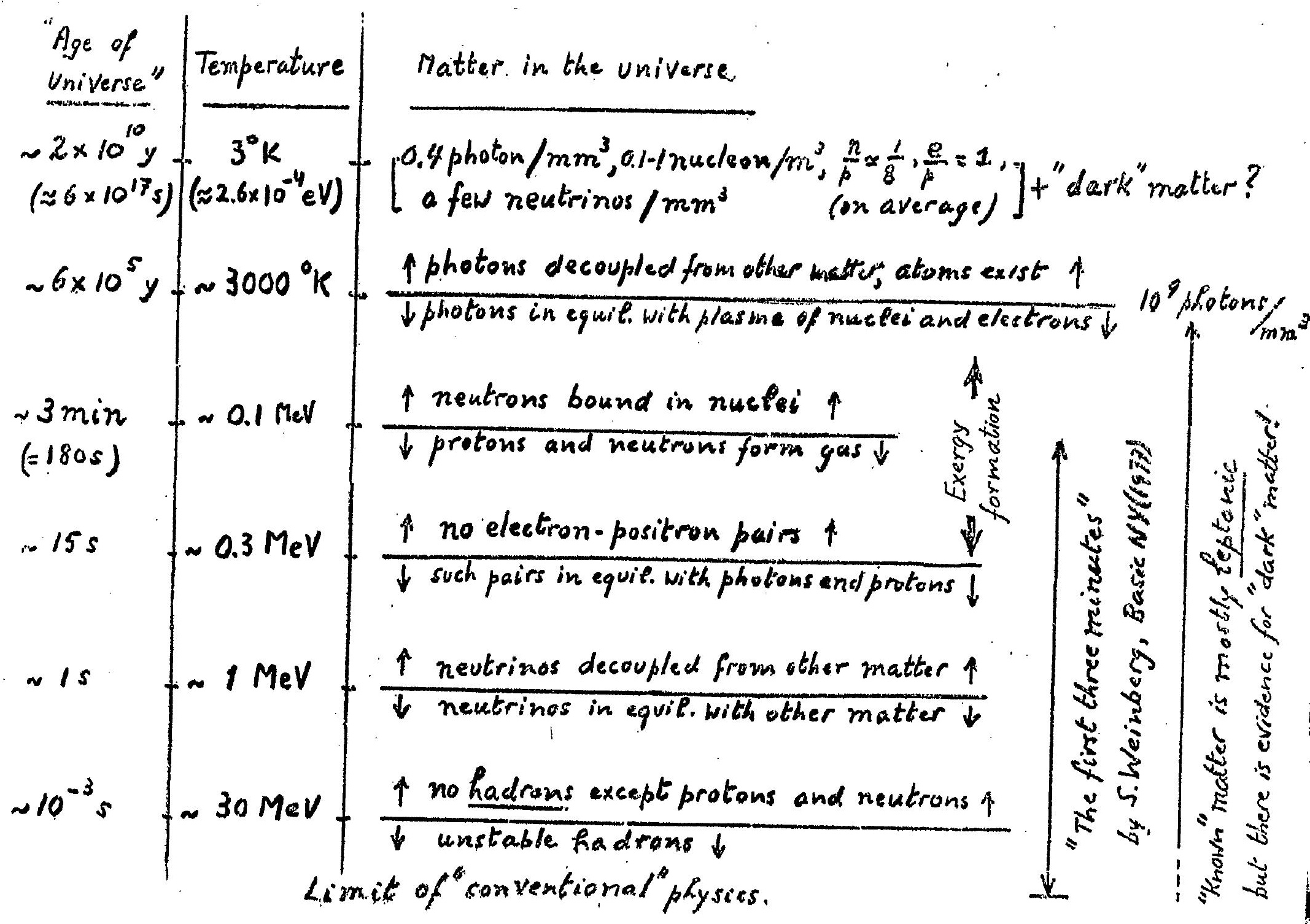


Pieture based on "zew" dfhysses and increasibgly speculative!"

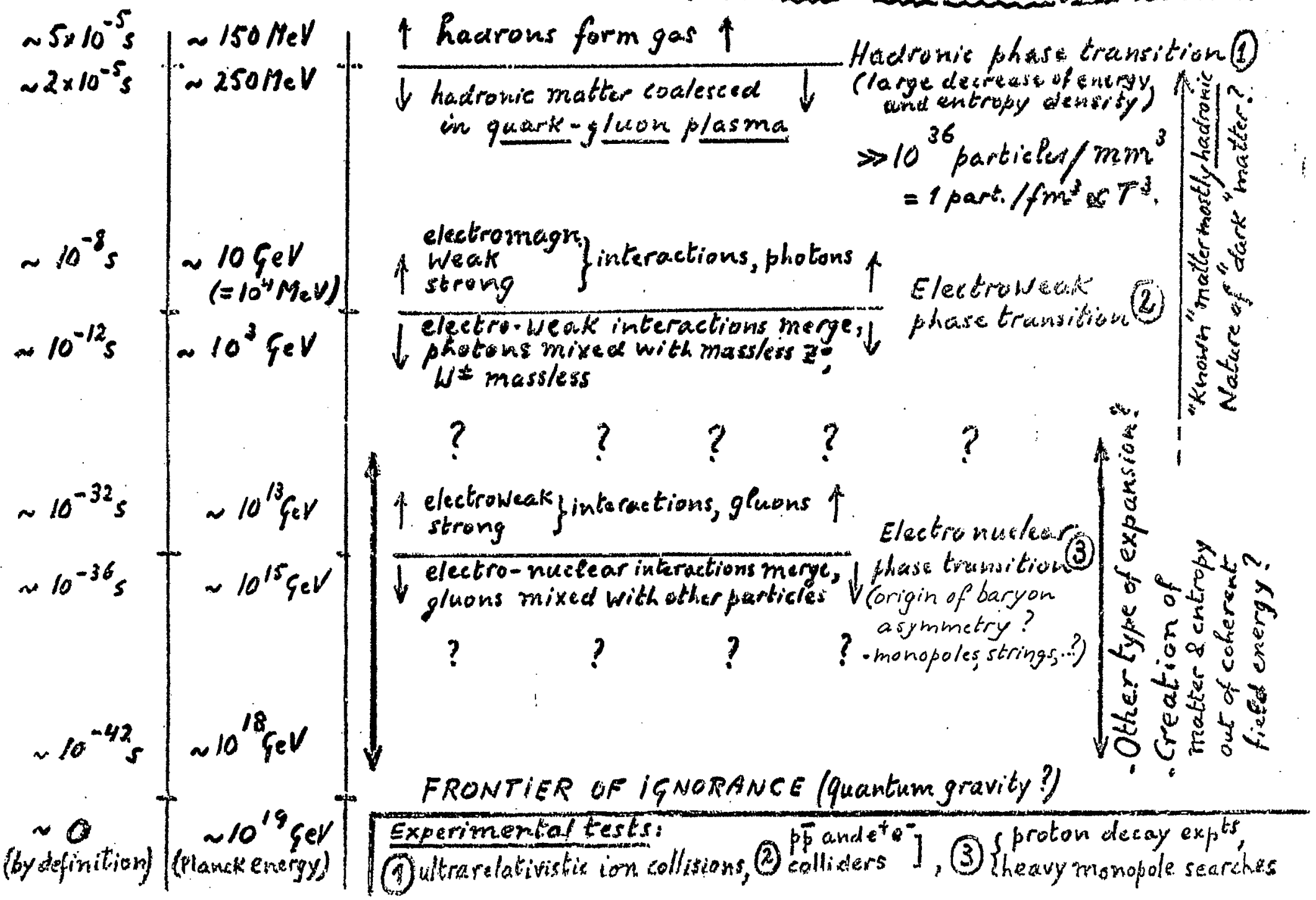


Basic equations for expanding Universe (homogeneous isotropic model)

- Metric : $d s^{2}=a^{d} t^{2}-d t^{2}, d t^{2}=a^{2}(t)\left[\frac{d r^{2}}{1-k r^{2}}+r^{2}\left(\theta^{2} \theta^{2}+\sin ^{2} \theta d d^{2} t_{j}^{2}\right)\right.$

$r, \theta, \varphi$ : space coordinates of commoving observers

$t$ : proper time of commoving observers

$d l$ : space distance, $a(t)$ scale parameter

$K$ : curvature constant $(k=0$ flat space, open

$k<0$ hyperbolic space, open life.".

$k>0$ spherical space, closed)

- Einstein equations for gravitation give:

$$
\left(\frac{t}{2} \frac{d a}{d t}\right)^{2}=\frac{8 \pi}{3} \xi_{f}-\frac{k}{a^{2}}+1
$$

cosmological constant - curvature term

gravity. attraction term, $q=$ Newton constants $p=$ hon-gravitational energy density

"kinetic energy term."

$$
\begin{aligned}
& \zeta^{1 / 2}=1.6 \times 10^{-33} \mathrm{~cm}\left(=\frac{t}{7}\right)=0.54 \times 16^{-43} \mathrm{sec}\left(=t_{T}\right)
\end{aligned}
$$

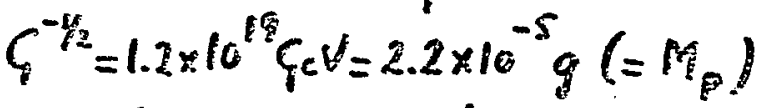

(for Planck, $K=c=0$ )

- Energy conservation for co -moving volume $V$.

$$
\frac{d}{d t}(\rho v)+f \frac{d t}{d t}=0 \Rightarrow \frac{d}{d t}\left(f \alpha^{*}\right)+\frac{d Q^{2}}{d t}=0
$$

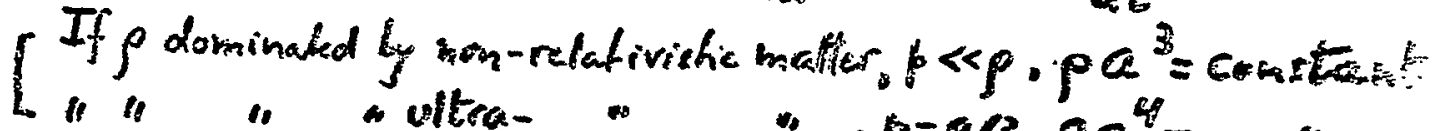

(po m-relativistic: $5 \times 1 c^{5} y \leq t \leq 1.5-2 \times 10^{10} y=$ today, ultra- " before)

At every time, state of mather is characterized by the particle species present and their equation of state. 

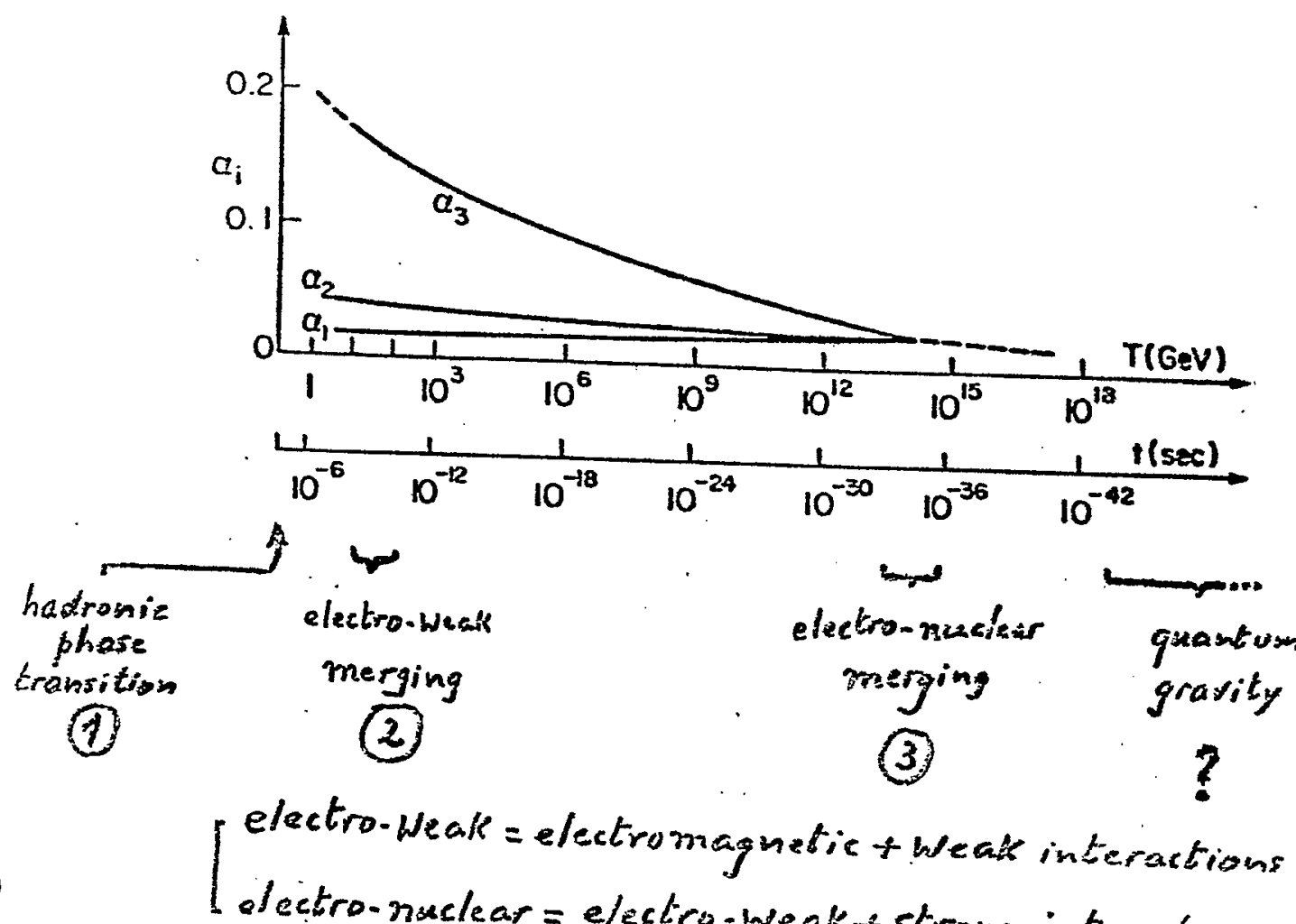

adronit

transition

(1)

II 
Hadrosic phase transition in the

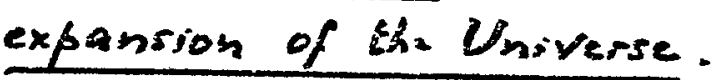

I
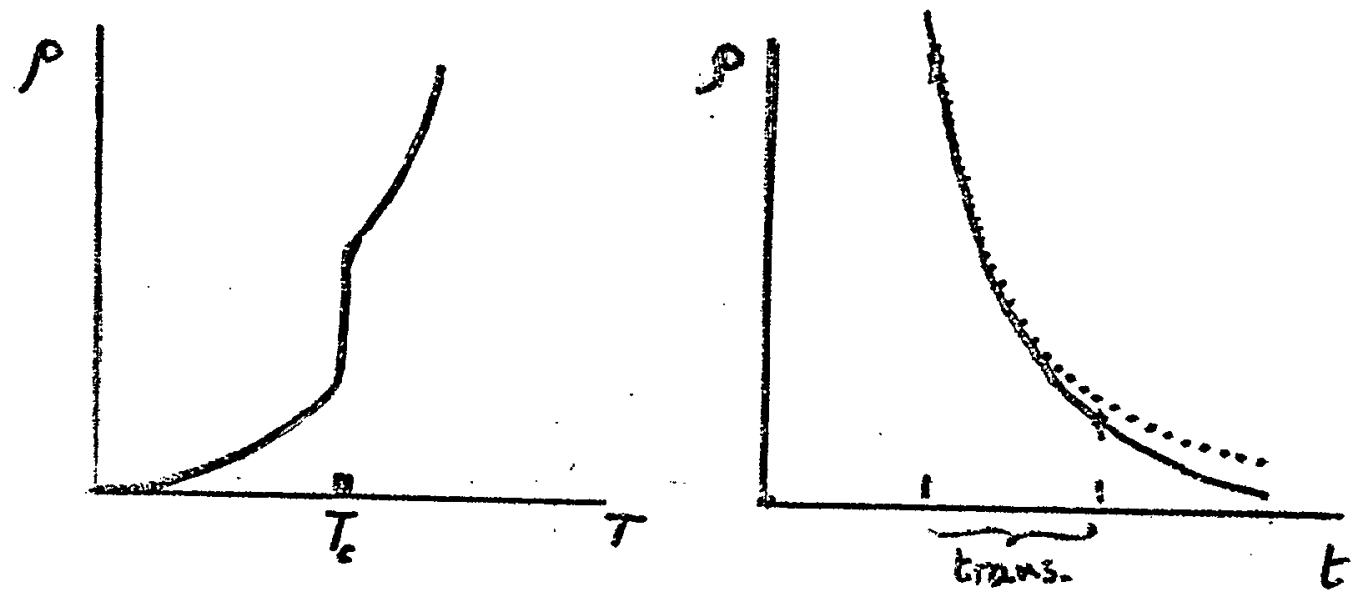

$\left(\frac{d}{a} \frac{d a}{d t}\right)^{2}=\frac{8 \pi}{3} \zeta_{p} \quad \frac{d}{d t}\left(\rho a^{3}\right)+p \frac{d a^{3}}{d t}=0$

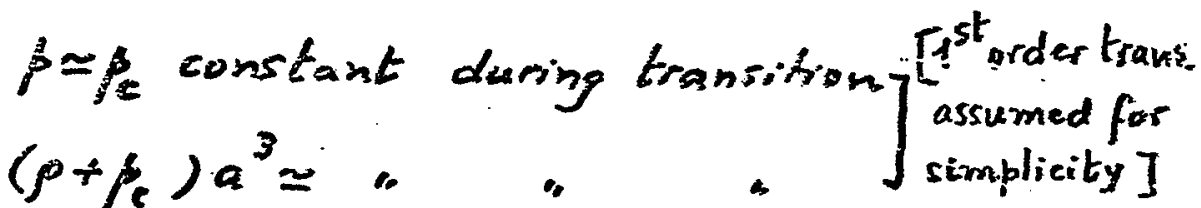

$$
\begin{aligned}
& \frac{d \rho}{d t}=-(24 \pi g)^{1 / 2} \rho^{1 / 2}\left(\rho+\beta_{c}\right) \\
& p \simeq \beta_{c}\left(\frac{\sqrt{p}-\sqrt{p_{c}} \tan y\left(t-t_{1}\right)}{\sqrt{p_{c}}+\sqrt{\rho_{1}} \tan y\left(t-t_{0}\right)}\right)^{2}
\end{aligned}
$$

$\left.y=\left(6 \pi C_{s}\right)_{s}\right)^{3 / 2}, t=$ beginning of transition, $t, \sim 10^{-5} s=3 \mathrm{~km}$ when $p\left(t_{1}\right)=p_{1}=\rho\left[Q \zeta P+\right.$ leptons $\left.+y^{\prime} s\right] a t T_{c}, \beta_{c}$.

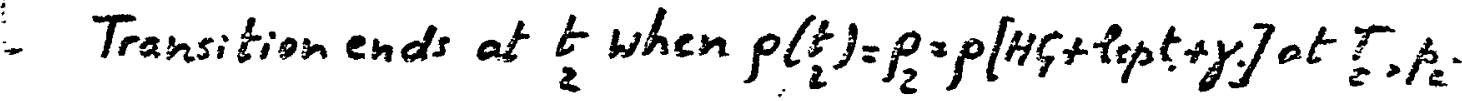
$P_{2} \sim 0.5-0.7 P_{1}, t_{2} \sim 1.2-1.5 t$.

At $t=t_{1}+f_{t}$, HC bubbles separated by $t_{1}^{1 / 3}(1 \mathrm{fm})^{2 / 3} \sim 10^{-7} \mathrm{~cm}$.

After percolation spaces structure may reach $t_{1}^{2 / 3}\left(1 \mathrm{fm}_{\mathrm{m}}\right)^{1 / 3} 1 \mathrm{~cm}$ 
Most probably, no trace is left torbay of the

VI hactronit phase transition in the early viniverse

But one cannot exelusk Some exotic possibilities:

1) Black hole formation (Schramin et al.) If $Q C^{P}$ regions of sage wt, surrounded by larger Hf regions wont form, some of them could collapse into blats holes of mass $\leq 10^{58} \mathrm{GCV}$ and radius $\approx 10 \mathrm{~km}\left(M_{0}=2 \times 10^{33} g=10^{57} \mathrm{geV}\right)$

The black holes cowls later contribute to galaxy formation.

They would how form invisible matter

(7) Crawford (D.N. Sehramm, Nature 298 (1981) 532; K.Freese, R. Price a 3.N. Schramm, Ap.J. 275 (1983) 405)

For this to work, the $\rho_{B H}$ in the black holes should now be $\sim(10-100) \rho_{\text {visible }}$

2) Formation of "strange punts" matter" (Whiten): a possible we w form of stable matter containing "1,5 quarts, could also form invisible matter in the present Universe

(E. Witter, Phys. Rev. D 30(1984)272)

There is something fascinating about Science One gets uhoinsale returns of conjecture out of such trifling investment of fact! (Mark Twain) 


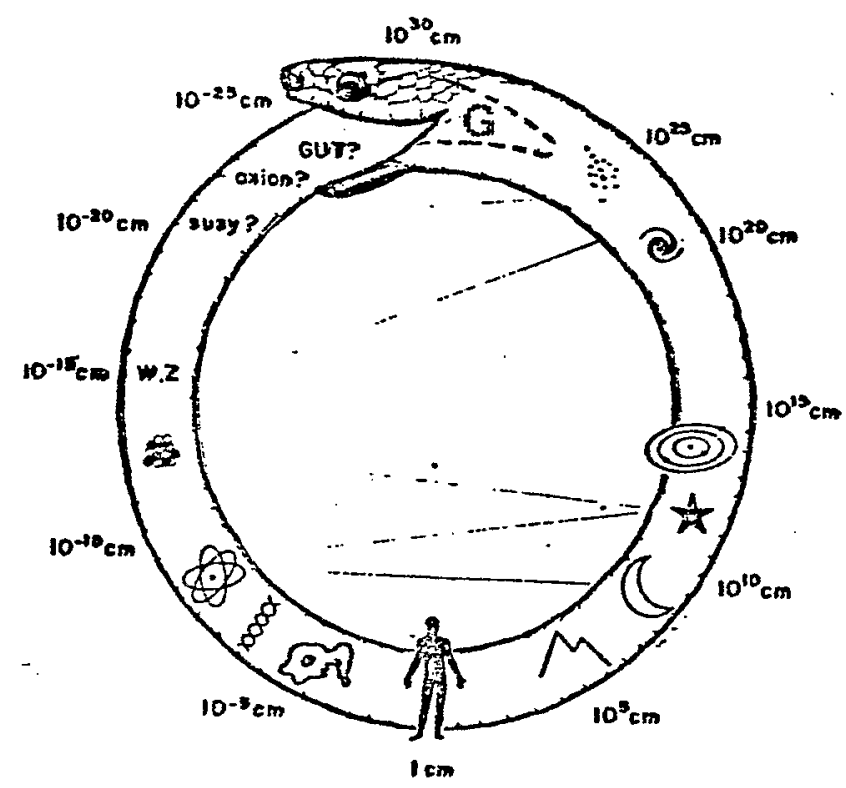

Fig. 7. New ideas in particle physics suggest further linkages between "micro" and "cosmic" scales (i.e. between left and right on this plcture) in addition to the well-known ones between atomic and nuclear physics and the terrestrial and stellar scales. The ultimate unification will involve quantum gravity (the Planck scale) and cosmology. (from Primack and Blumenthal, Proc. 3rd Horiond Astrophysics Workshop (Reidel) p. 180 (1983)). 